\title{
Development of Top-Up And Bottom -Up Techniques for Assessment of Power Line Communication Channel Model
}

\author{
Ganiyu Adedayo Ajenikoko Babajide Akinjobi \\ Department of Electronic and Electrical Engineering, Ladoke Akintola University of Technology, P.M.B, 4000, \\ Ogbomoso, Nigeria
}

\begin{abstract}
Power Line Communication (PLC) is the transfer of data and voice signals from one communication system to another over the electric power delivery network. However, with the advent of technology, human dependency on electricity and communication has grown beyond leaps and bounds. The power transmission line channel has not been designed with wired channel requirements for broadband applications and appears as a harsh environment for the low-power high-frequency communication signals. This study therefore evaluate the performance of PLC on power grid by simulating a practical multipath power line communication channel model using top-down and bottom-up approach. The statistical multipath parameters such as path arrival time, magnitude and interval for each category were analyzed. This is done over the frequency range of $100-300 \mathrm{kHz}$. The result shows that at $100 \mathrm{kHz}$, data can be transmitted up to 350 meters without signal distortions while for $300 \mathrm{kHz}$ only 50 meters can be covered. In addition, signal attenuation with a distance of $2 \mathrm{~km}$ is about 0.2 of the original signal at $300 \mathrm{kHz}$ and less at higher frequencies. The results in the time and frequency domains indicate that data transmission in PLC environment needs signal to be amplified at higher powers.
\end{abstract}

Keywords: Power Line Communication, Power Grid, Transmission Line, Multipath Parameters, Channel Modelling, Top-Down Approach, Bottom-Up Approach.

DOI: $10.7176 / \mathrm{JNSR} / 9-16-03$

Publication date: August $31^{\text {st }} 2019$

\section{I.INTRODUCTION}

Power line Communication (PLC) is an alternative method for broadband networking that has the advantage of transmitting over channels already used for electrical distribution or transmission [1]. PLC has become one important option for the required data communications infrastructure and alternative for conventional wired and wireless in-door data transmission due to the development of robust modulation, channel coding, and digital signal processing technologies. Power Line Networking enables the implementation of Local Area Networks (LANs) and fast internet amongst other applications. The broadband PLT operates in frequencies from $150 \mathrm{kHz}$ to $34 \mathrm{MHz}$ having a theoretical maximum speed of $200 \mathrm{Mbps}[2,3]$.

The concept of communicating through PLC is quite old but not brought into use on massive scale for commercial purpose. Power companies have been using this service and keeping it restricted to them only [2]. There are several reasons that hinder PLC from being chosen as communication medium. The first and foremost reason is that power reaches the user from the point of generation via three different voltage level (HV, MV, LV). At the same voltage level, PLC allows communication while maintaining the quality well above the minimum threshold $[2,4,5]$.

Another disadvantage of PLC is that a data signal injected to power line could not pass through transformer. The use of bypass devices across transformers increases the complexity and adds to overall cost [3]. Data signal is separated before the transformer instead of going through it and again injected back to the power line. Significant transmission and distribution loss of power lines is another characteristic which makes PLC a secluded option. A clique is produced when a device is switched ON or OFF in a network [2]. The Impulsive noise depletes the signal quality by introducing noise in the system. As power lines are not insulated, at high frequencies they act as an antenna hence interfering with signal being generated from high tension wire in close vicinity $[4,6,7]$.

The increase in electrical supply networks informed the rapid evolution of the PLC technology and the ever increasing demands in the area of communications [5,6]. The development of accurate PLC channel transfer characteristic models is very important as it forms the basis for computer simulations which are useful in appropriate system design and further enabling the analysis of the performance of different schemes as well as recognizing probable difficulties in the development of communication systems in different network configurations and loads $[8,9]$.

A PLC system is illustrated in Figure 1 where the signal tranverses from the transmitter to receiver through couplers [10]. The power line channel is mainly characterized in terms of the attenuation parameter and the impedance parameter which arises from mismatches in the power line network. The noise parameter, which is added to the signal, originates from several sources. A coupling circuit is used to connect the communication system to the power-line [11]. The purpose of the coupling circuits is inn two-folds. Firstly, it prevents the 
damaging $50 \mathrm{~Hz}$ signal, used for power distribution to enter the equipment. Secondly, it certifies that the major part of the received/transmitted signal is within the frequency band used for communication. This increases the dynamic range of the receiver and makes sure the transmitter introduces no interfering signals on the channel [2, $12,13]$.

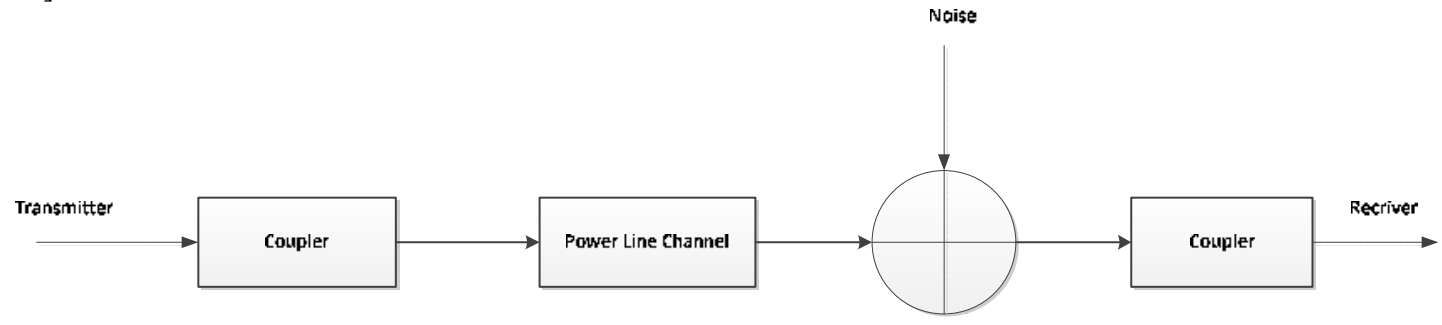

A. Noise in PLC

Figure 1: Simplified Power Line Communication System

The performance and reliability of the PLC network is affected by noise. The low-voltage (LV) power lines are a power transmission cable connecting substations to domestic houses [12]. The source of noise at Low-Voltage (LV) which is a power transmission cable connecting substations to domestic houses can be internal (inside the power network) or external (outside the power network). The noise in PLC network is classified as [13, 14]:

i. Coloured Background Noise: Is the mostly generated sources at noise of low intensity. They are characterized by a power spectral density that decreases with frequency. Appliances and components operating at low power, collectively generates noise with relatively low power spectral density (PSD).

ii. Narrow-Band Noise: Is mostly in sinusoidal form or modulated signal with various origins, and generated by the existence of broadcast waves and the instabilities caused by appliances with a transmitter or receiver.

iii. Impulsive Noise: Is considered as the most significant, and is mostly generated by electrical appliances connected to the network. It is further classified as;

a. Periodic Impulsive Synchronous to the mains, which is generated by power supplies with silicon controlled rectifiers. Components like rectifier diodes and transistors whose cut off voltage and threshold voltages lead to switching actions.

b. Periodic Impulsive Synchronous to the mains, which is mostly caused by switched-mode power supplies.

c. Asynchronous Impulsive noise which is caused by switching transients in the power network.

Collective noise is the sum of all the noise types mentioned above. Colored Noise and Narrow Band Noise are considered as background noise which uniformly spread throughout the spectrum, as the rate of change of magnitude is very slow. Background noise is considered to be Additive White Gaussian Noise (AWGN) $W k$ for PLC assessment. The impulsive noise is given by $[12,15,16]$ :

$$
i k=b k * g k
$$

where, $b k$ is the Poisson process which is the arrival of the impulsive noise, $g k$ is the white Gaussian process with mean zero and variance $2 \sigma^{2}$. That is Gaussian noise of magnitude varying up to $35 \mathrm{~dB}$ and is distributed among data bits complying Poisson distribution. $b k$ is the probability of getting it by noise and $g k$ is the random variable denoting the varying amplitude of noise. The total noise $n k$ is given by $[11,13,17,18]$ :

$$
\begin{aligned}
& n k=W k+i k \\
& n k=W k+b k * g k
\end{aligned}
$$

Arrival of the impulsive noise follows the Poisson process with a rate of $\mathrm{R}$ units per second, so that the event of $\mathrm{k}$ arrivals in $\mathrm{t}$ seconds has the probability distribution as $[17,18]$ :

$$
p k t=e-\lambda t-\lambda t k / k \text { ! }
$$

Let $a k$ be the received signal, and then the transmitted signal $r k$ is given by [19]:

$$
r k=a k+n k
$$

\section{B. Multipath Channel Model of Power Line Communication}

In Power line transmission the propagation of data signals do not follow single path or uni-path, but a multipath pattern similar to wireless signals that involves cellular transmission [20]. Power grid with Lower Voltage (LV) is a single central transmission line with shooting stems terminating at the end users place, as shown in Figure 2. $T X$ is the point of transmission (substation/service provider) and $R X$ is the point of receiver (automated meter, customer or other appliances) [12]. A small section of Figure 3 could be singled out to review multipath of propagation of signal. From Figure 3 [20]; 
Let $\mathrm{D}$ be the point of transmission and $\mathrm{C}$ be the point of receiving. The signal generated at point $\mathrm{D}$ is given as [17]:

1. D $-3-2-\mathrm{C}$

2. $\mathrm{D}-3-3-\mathrm{D}$

3. $\mathrm{D}-3-1-3-\mathrm{D}$

4. $\mathrm{D}-3-1-1-2-\mathrm{C}$

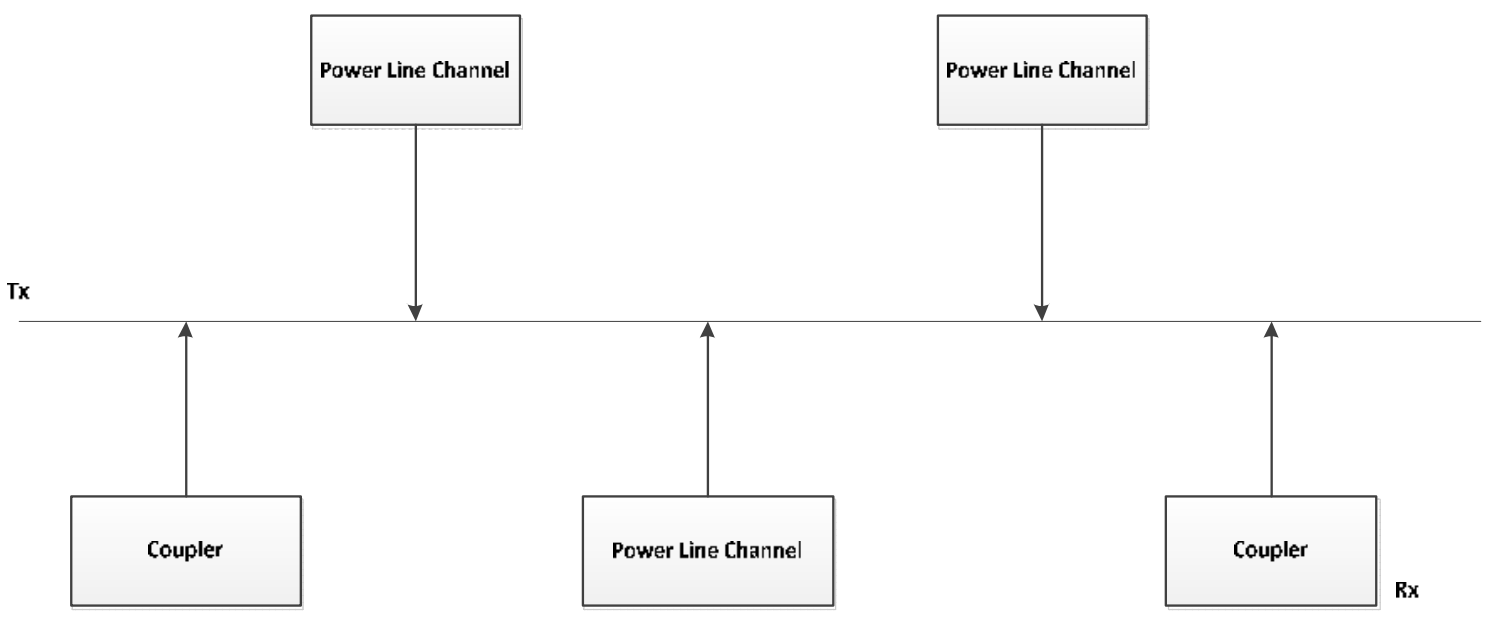

Figure 2: Typical topology of end a transmission line in power grid

D

(3)

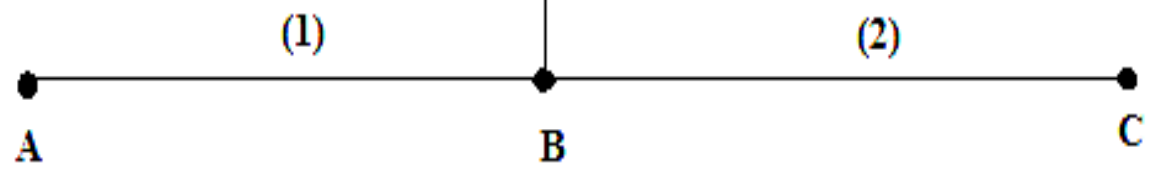

Figure 3: Multipath propagation of signal from D to C

\section{MATERIALS AND METHOD.}

This study used top-down and bottom-up approach to measure the channel characteristics of the power line under different network topologies and conditions.

For top-down approach, the model parameters are obtained from measurements. Little computation is necessary in this approach and it is easy to implement. The transfer function parameters are extracted from actual measurements of the given PLC channel. The transfer function for the channel is given as:

$$
H(f)=\sum_{i=1}^{N} \rho_{i} \cdot e^{-j 2 \pi f t_{i}}
$$

Where;

$N$ is the number of signal flow paths

$i$ is each flow path

$t_{i}$ is the time delay

$\rho_{i}$ is the complex factor which is the product of transmission and reflection factors.

The complex attenuation factor is given as: 


$$
\rho_{i}=\left|\rho_{i}\right| \cdot e^{-j \varphi_{i}}
$$

where;

$$
\varphi_{i}=\tan ^{-1}\left(\frac{\operatorname{Im}\left(\rho_{i}\right)}{\operatorname{Re}\left(\rho_{i}\right)}\right)
$$

The Multipath Model channel transfer characteristic by a frequency response is given as:

$$
H(f)=\sum_{i=1}^{N} g_{i} \cdot e^{-\left(a_{0}+a_{1} f^{k}\right) d_{i}} \cdot e^{-j 2 \pi f \tau_{i}}
$$

Where;

$g_{i}=$ the weight of the $i^{\text {th }}$ path determined by reflections and transmission,

$d_{i}=$ the path length,

$\tau_{i}=$ the path delay given by the following

$$
\tau_{i}=\frac{d_{i}}{v_{p}}=\frac{d_{i} \sqrt{\varepsilon_{r}}}{c_{0}}
$$

Where;

$$
\begin{aligned}
& \varepsilon_{r} \text { is the insulating material's dielectric constant } \\
& c_{0} \text { is the speed of light } \\
& d_{i} \text { is the length of a path } \\
& v_{p} \text { is the propagation speed }
\end{aligned}
$$

For bottom-up approach power network elements are modeled mathematically and incorporated to generate the PLC channel. This approach can be applied to various situations flexibly as long as the network information is perfectly known. In addition, this approach is closely related to the physics of power networks since it is derived from the physical interpretation of Electromagnetic (EM) wave propagation in transmission line networks. For a given number of paths, an estimate of the path attenuation, weighting and delay factor are determined.

The Multipath Model of bottom-up approach is given as:

$$
H(f)=\sum_{i=1}^{N} g_{i} \cdot e^{-\alpha \cdot d_{i}} \cdot e^{-j 2 \pi f \frac{d_{i}}{v_{p}}}
$$

The PLC channel model is given as:

$$
H(f)=\sum_{i=1}^{N} g_{i} \cdot e^{-\left(a_{0}+a_{1} f^{k}\right)} \cdot e^{-j 2 f\left(\frac{d_{i}}{v_{p}}\right)}
$$

The transfer function is obtained by a Fourier transformation of the measured impulse response. This impulse response is represented as a sum of Dirac pulses delayed by $\tau_{i}$ and is expressed as:

$$
h(t)=\sum_{i=1}^{N}\left|\rho_{i}\right| \cdot e^{-j \varphi_{i}} \cdot \delta\left(t-\tau_{i}\right)
$$

The model is completed by assigning proper values to the above parameters. The intrinsic line parameters are derived prior to the calculation of the transfer function shown in equation (12).

\section{III.DISCUSSION OF RESULTS}

The assessment of PLC channel model is presented in Figure 4. The result was presented with frequencies channel responses of $100 \mathrm{kHz}$ and $300 \mathrm{kHz}$ respectively.

Figure 4 shows the relationship between the weight of the path and the path delay of PLC channel at frequencies of $100 \mathrm{kHz}$ and $300 \mathrm{kHz}$. The result indicated that the highest path delay value is 0.62 seconds at 250 weight value at $100 \mathrm{kHz}$ and $300 \mathrm{kHz}$. The lowest path delay values are -0.55 and -0.60 seconds at 750 weight value at $100 \mathrm{kHz}$ and $300 \mathrm{kHz}$ respectively. In general, the result shows that at $100 \mathrm{kHz}$, data can be transmitted up to 350 meters without signal distortions while at $300 \mathrm{kHz}$, only 50 meters can be covered. It can be observed that the signal attenuate with distances in such a way that at a distance of $2 \mathrm{~km}$ the signal is about 
0.2 of the original signal at $300 \mathrm{kHz}$ and less at higher frequencies. This behavior indicates that data transmission in PLC environment needs signal to be amplified or transmitted at higher powers.

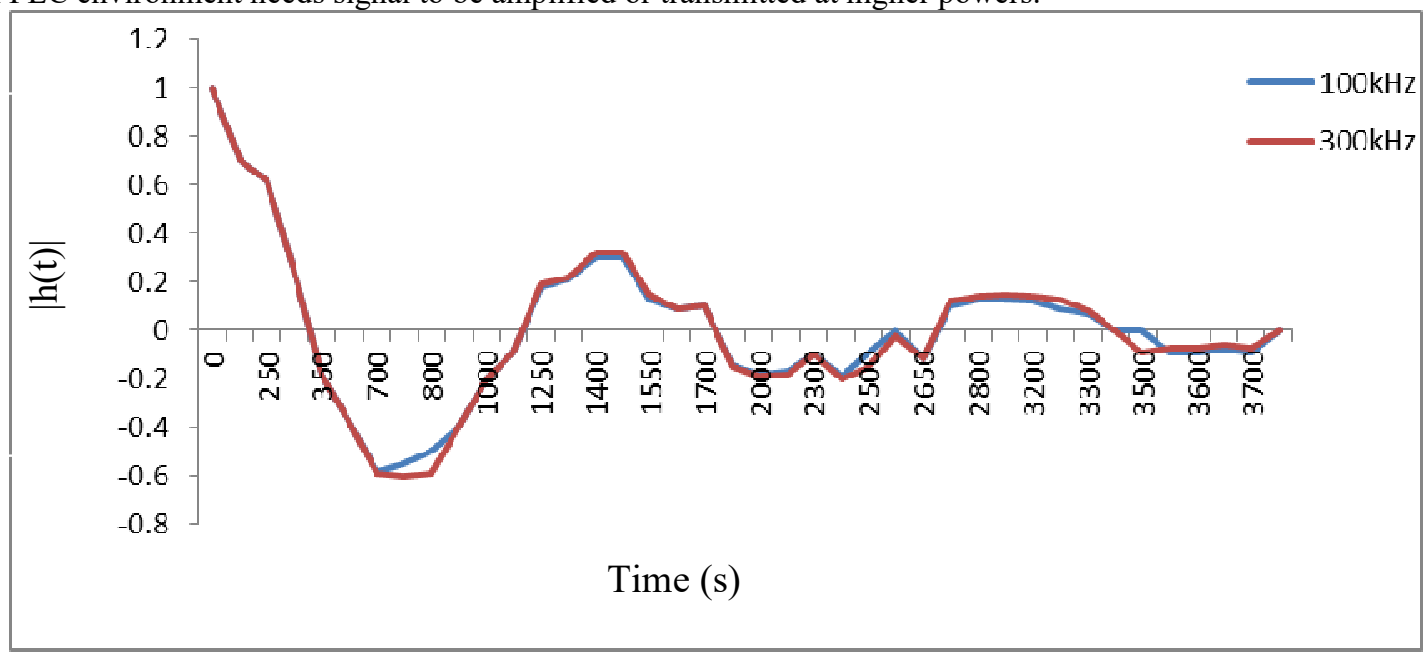

Figure 2: Time response of weight of the path and the path delay of PLC channel for $100 \mathrm{kHz}$ and $300 \mathrm{kHz}$ frequencies.

\section{IV.CONCLUSION}

This study has presented the assessment of power line communication channel model using both top-up and bottom-up communication techniques. In Power line transmission the propagation of data signals do not follow single path or uni-path, but they follow a multipath which is a pattern very similar to wireless signals involved in cellular transmission. This model is basically a multipath model partly cooperated with transmission line theory. The multipath nature of PLC channel is due to the presence of branches and impedance mismatches which cause multiple reflections. This study treats the PLC channel as a black box and a large number of measurements are collected by exciting the channel with a reference signal in either time domain or frequency domain.

The result shows that at $100 \mathrm{kHz}$, data can be transmitted up to 350 meters without signal distortions while at $300 \mathrm{kHz}$, only 50 meters can be covered. It can be observed that the signal attenuate with distances in such a way that at a distance of $2 \mathrm{~km}$, the signal is about 0.2 of the original signal at $300 \mathrm{kHz}$ and less at higher frequencies. The study revealed that there is an increase in power line communication length in deep notches which signifies attenuation on the broadband power line communication channel. The results indicated that the channel transfer function is effective for modelling the power-line communication channel.

\section{REFERENCES}

[1] Anastasiadou, D. and Antonakopoulos, T. (2005). "Multipath characterization of indoor power-line networks", IEEE Transactions on Power Delivery20 (1), 90-99.

[2] Anatory, J., Mvungi, N. H. and Kissaka, M. M. (2014). "Analysis of power line channel model for communication from primary substation node to end-users", Iranian Journal of Electrical and Computer Engineering, 3(1), 9-15.

[3] Carlson, A. B., Crilly, P. B. and Rutledge, J. C. (2002). "Communication systems, an introduction to signals and noise in electrical communications", McGraw Hill, 2002, 618-620.

[4] Cortés, J. A., Sanz, A., Estopiñán, P. and García, J. I. (2015). "Analysis of narrowband power line communication channels of an advanced metering infrastructure", EURASIP Journal on Advances in Signal Processing (2015):27, 1-13.

[5] Guillen, E. P., López, J. J. and Barahona, C. Y. (2008). "Throughput Analysis over Power Line Communication Channel in an Electric Noisy Scenario", World Academy of Science, Engineering and Technology, International Journal of Electronics and Communication Engineering, 2, (7), 1344-1350.

[6] Hashmat, R., Pagani, P., Zeddam, A. and Chonave, T. (2011). "A channel model for multiple input multiple output in-home power line networks", IEEE International Symposium on Power Line Communications and Its Application, 35-41.

[7] Jae, Y. P., Choong, S. H., Sungwon, L. (2011). "An efficient MAC scheme with modified RTS/CTS of IEEE P1901," Int. Conference Information Networking (ICOIN), 230-234.

[8] Llano, A., Angulo, I., Angueira, P., Arzuaga, T. and Vega, D. (2016). "Analysis of channel influence on power line communications based on ITU-T G.9904 (PRIME)”, Energies 2016, 9, 39; doi:10.3390/en9010039, 9-39. 
[9] Paul, C. (2008). "Analysis of multiconductor transmission lines", 2nd Edition, John Wiley and Sons, New Jersey, 2008

[10] Sagar, N. (2011). "Power line communications systems: overview and analysis", A thesis submitted to the Graduate School-New Brunswick Rutgers, The State University of New Jersey In partial fulfillment of the requirements For the degree of Master of Science Graduate Program in Electrical and Computer Engineering, New Brunswick, New Jersey, 1-160.

[11] Sartenaer, T. and Delogne, P. (2006). "Deterministic modeling of the (shielded) outdoor power line channel based on the multiconductor transmission line equations", IEEE Journal on Selected Areas in Communications 24 (7), 1277-1291.

[12] Singh, V. P. (2012). "Analysis of power line communication channel model using communication techniques", Master of Science in Electrical \& Computer Engineering, North Dakota State University, $1-49$.

[13] Tonello, A. M. (2007). "Wideband impulse modulation and receiver algorithms for multiuser power line communications, EURASIP Journal on Advances in Signal Processing, 1-16.

[14] Tonello, A. M., Versolatto, F., Bejar, B. and Zazo, S. (2012). "A Fitting Algorithm for Random Modeling the PLC Channel”, IEEE Transactions on Power Delivery 27 (3) (2012) 1477-1484.

[15] Varma, M. K., Jaffery, Z. A. and Ibraheem, K. (2019). "Broadband power line communication: the channel and noise analysis for a power line network", International Journal of Computer Networks \& Communications (IJCNC), 11(1), 81-92.

[16] Veronesi, D., Riva, R., Bisaglia, P., Osnato, F., Afkhamie, K., Nayagam, A., Rende, D. and Yonge, L. (2011). "Characterization of in-home MIMO power line channels", IEEE International Symposium on Power Line Communications and Its Applications, IEEE, 2011, pp. 42-47.

[17] Yin, Y. (2017). "Application of PLC Control System and Communication Technology in Chemical Plant Equipment Renovation", Chemical Engineering Transactions, 62, 679-684.

[18] Yousuf, M. S., Rizvi, S. Z. and El-Shafei, M. (2008). "Power line communications: an overview-part ii," ICTTA Int. Conference Inform. And Commun. Technologies: From Theory to Applications, 1-6.

[19] Zimmermann, M. and Dostert, K. (2002). "A multipath model for power line channel," IEEE Trans. Commun., (50), 553-559.

[20] Zwane, F. (2014). "Power line communication channel modelling", Dissertation Submitted in Fulfilment of the requirements for the degree Master of Science in Engineering: Electronic Engineering in the College of Agriculture, Engineering and Science, University of Kwazulu-Natal, 1-92. 